thermal error modelling, large scale metrology, virtual climatization

\author{
Dominik EMONTS ${ }^{1 *}$ \\ Mark P. SANDERS ${ }^{1}$ \\ Benjamin MONTAVON ${ }^{1}$ \\ Robert H. SCHMITT ${ }^{1,2}$
}

\title{
MODEL-BASED, EXPERIMENTAL THERMOELASTIC ANALYSIS OF A LARGE SCALE TURBINE HOUSING
}

\begin{abstract}
Temporally and spatially unstable thermal conditions lead to inhomogeneous thermoelastic changes in the workpiece geometry. Consequently, non-negligible geometric deviations are evident, especially when measuring large workpieces with narrow tolerances, which often take place in non-climatized production environments and thus make thermal monitoring indispensable. Accurate determination of the thermoelastic behaviour for complex and large geometries is a challenging task with computationally effortful or less accurate existing solutions. Thus, the development of innovative measurement and modelling approaches is subject of current research, whereat physical validation is a prerequisite. Therefore, the authors developed a method, enabling the emulation of typical process heat cycles on a turbine housing in combination with a geometric measurement system. The idea is to provide reproducible and reversible thermal conditions on a representative large workpiece and to investigate the resulting geometric deformation in an economically viable way. Throughout this study, an analogy test rig is presented, integrating different temperature sensors, two geometric measurement systems and thermal deformation models into one demonstrator. The demonstrator's first applications show insightful results, revealing accordance, but also unexpected deviations between the predicted and measured quantities. Moreover, it provides great potential for validation of more complex modelling approaches and innovative thermal condition monitoring systems for large precision workpieces.
\end{abstract}

\section{INTRODUCTION}

Thermal influences can account for a majority of errors in precision manufacturing. Therefore many research activities are dedicated to mitigate such effects [1-4]. Temporarily changing workpiece temperatures highly influence final part quality in production or accuracy in measurement processes [5-7]. Hence, temperature is included in multiple metrology standards, e.g. ISO 15530-1 and ISO 10360-2 for CMMs [8, 9]. For precise measurements, preventing thermal influences by measuring inside a temperature-controlled chamber and tempering the workpiece before measurement is the gold standard [10,11]. Especially for large workpieces driving energy transition, e.g. wind, gas or hydrogen turbines, where

\footnotetext{
${ }^{1}$ Laboratory for Machine Tools and Production Engineering (WZL) of RWTH Aachen University, Chair of Production Metrology and Quality Management, Aachen, Germany

${ }^{2}$ Fraunhofer Institute for Production Technology IPT, Aachen, Germany

*E-mail: d.emonts@wzl.rwth-aachen.de https://doi.org/10.36897/jme/146435
} 
geometric inspection takes place in non-controlled environments, thermal workpiece effects can significantly contribute to measurement uncertainty. This effect is leveraged by relatively small ratios of tolerance and nominal dimension of about 1:50,000 and higher considering workpiece sizes of up to $15 \mathrm{~m}$. [7]

Availability and practicability of calibrated workpieces for measurement correction (see ISO 15530-3 [12]) is limited for large workpieces. While steady state linear thermal expansion can be compensated for, also involving errors due to uncertainty in thermal expansion, environmental temperature variation is an additional uncertainty source [10]. The same applies to internal heat and thermal gradients within the workpiece while being more difficult to quantify at all. In terms of tolerance management, a high share of unknown systematic measurement deviations must be expected, which have to be estimated conservatively and thus can decrease overall manufacturing tolerance. Spatial thermal gradients within the workpiece increase in relevance with growing workpiece size, mass and precision requirements. This is particularly challenging for on-machine measurements, where geometric inspections are performed in shopfloor environments and cycle times between manufacturing and measurements are shortened to drive operational efficiency $[13,14]$. Thus, stored heat and thermal exchange with the environment is dynamic and difficult to quantify.

The following paper describes an experimental setup aiming to quantify aforementioned effects with an exemplary test workpiece. It thereby addresses the need for a sustainable, reproducible solution to systematically investigate thermoelastic deviations without reject production of costly, resource intensive parts, and illustrates a challenge in shopfloor measurements of large workpieces.

\section{THERMOELASTIC WORKPIECE MODELLING}

The state of the art regarding thermoelastic workpiece compensation for the characteristic evaluation of measurement deviations is limited [7]. Existing approaches are mainly composed of three-dimensional linear scaling or the usage of calibrated workpieces whereat steady, homogeneous temperature distributions are assumed [15].

Further improvements on traditional scaling consider selection, positioning, and weighting of individual temperature sensors, whereas supplemental refinements address the transformation for different sub-parts of features of a measurand. Separate transforms, derived for each section, can then be interpolated across the entire measurand. [6]

More complex solutions, e.g. FEM, can reach superior accuracies, but suffer from high computational efforts for large problems and often uncertain initial and boundary conditions describing thermal and mechanical loads on the workpiece. FEM approaches are used in particular for chipping process simulations [16-19]. As thermal boundary conditions can be undetermined and change during the inspection process in thermally uncontrolled environments they must be incorporated into the model, which is a non-trivial task. An upcoming branch of research aims to combine scientific knowledge of physics-based models with neural networks to leverage the output of physics-based model simulations along with observational features, which can be provided by sensors, in a hybrid modeling setup to generate predictions [20]. 
Considering the simulation of thermoelastic workpiece behavior during measurements, the initial and boundary conditions are almost the same as for machining processes. They may cover ambient temperature profiles, initial workpiece temperature distributions as well as heat transfers besides mechanical loads, such as gravity or fixing. Thus, physical modelling includes unsteady, three-dimensional heat conduction with convection and radiation coupled with thermal expansion [21]. For measurement processes overall temperature changes and gradients are generally smaller than for machining processes, hence small environmental condition variations are more relevant than for machining processes.

\section{CONCEPTION OF THERMAL TEST RIG}

Testing is a key element in the development of engineering methods and products. There are three basic types, which can be broken down into field testing, analogy testing and simulation. Field testing is generally considered to be the most accurate and elaborate method. Its significance can be explained by the fact that product tests are performed in the actual context in which it will be used. Thus, it is the most time and resource consuming method. Especially in large scale manufacturing, field testing can be very elaborate and cost-intensive due to high unit costs, expensive machine times and significant handling efforts.

Analogy testing, as cost-effective alternative, seeks to represent the reality by means of abstracted test rigs, which can generate realistic results and at the same time be less burdensome and resource-consuming. Simulation, as the third and most recent technology, foregoes physical testing and emulates real testing by using computational power. Thus, it is usually the fastest and cost-efficient way. Nevertheless, it is based on algorithms fed with multiple, often estimated parameters, which highly affect the accuracy of results. Therefore, simulation parameters must be set carefully, and results validated against real measurements.

In this paper an analogy test rig is presented, enabling reversible heating and cooling cycles on a turbine housing using heat pads as external heat sources and measure dimensional displacements over changing temperature profiles in parallel. The setup emulates heat fluxes on functional surfaces typically coming from a localized chipping process and thus aims at the systematic investigation of the thermoelastic behavior of a large workpiece in a shopfloor environment.

\subsection{MENT SYSTEMS AND PROOF OF SUITABILITY}

Throughout this study, two different testing setups were implemented. The first testing setup is illustrated in Fig. . It is comprised of four major components, namely: Turbine housing, mobile coordinate measurement machine (mCMM), heat pads and controller. Since high surface roughness of untreated cast iron adversely affects the uncertainty of tactile measurements, counterbores were drilled for dedicated positions (B1, B13, B24). The essential characteristics of utilized components can be inferred from Table. Blue marked dots (see Fig. ) represent wall-incorporated sensors, which monitor temperature distributions over 
respective wall cross-sections. For any position, three temperature sensors are equidistantly distributed for the different height dependent wall thicknesses, varying between $32 \mathrm{~mm}-$ $70 \mathrm{~mm}$.

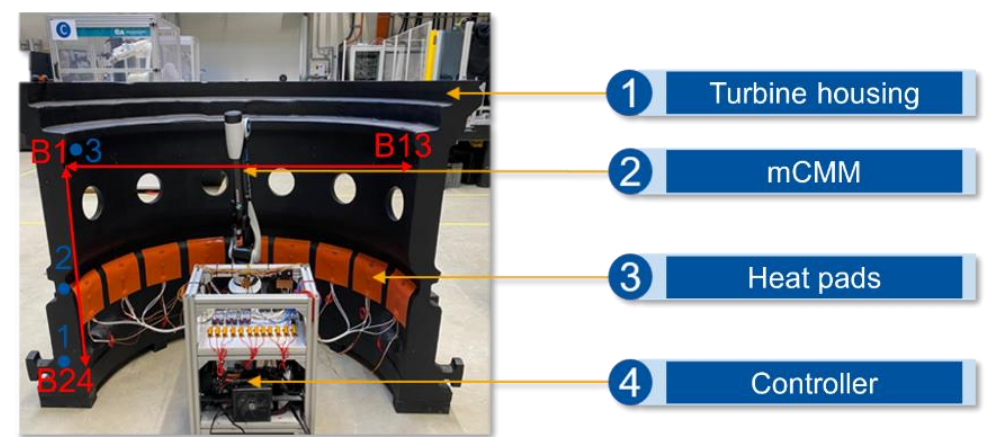

Fig. 1. Experimental setup with mCMM. Lines show mCMM measurements. Blue dots represent radially distributed temperature measurement points (see Fig. 2)

Table 1. Experimental setup components. 2.1 is used with the first experiment, 2.2 with the second experiment

\begin{tabular}{|c|c|c|}
\hline & Component & Characteristics \\
\hline 1 & Turbine housing & $\begin{array}{l}\text { Material: Cast iron C45E, CTE (literature): } 11.1 \mathrm{ppm} / \mathrm{K} \text {, } \\
\text { Weight: } 1500 \mathrm{~kg} \\
\emptyset_{\text {inside }}=1425 \mathrm{~mm} \\
\text { A: } 7000000 \mathrm{~mm}^{2}\end{array}$ \\
\hline 2.1 & $\mathrm{mCMM}$ & $\begin{array}{c}\text { Type: Hexagon Absolute Arm } 85 \\
M P E=23 \mu \mathrm{m}\left(U_{M P E}=M P E / \sqrt{3}=13.3 \mu \mathrm{m}\right)[22] \\
\text { range: } 2000 \mathrm{~mm}\end{array}$ \\
\hline 2.2 & Laser tracker & $\begin{array}{c}\text { Type: API Radian } \\
M P E=28 \mu \mathrm{m}\left(U_{M P E}=16.2 \mu \mathrm{m}\right)\end{array}$ \\
\hline 3 & $9 \times$ Heat pads & $\begin{array}{c}\text { Dimensions: } 200 \times 200 \times 2 \mathrm{~mm}^{3} \text {, Voltage: } 12 \mathrm{~V} \text {, } \\
\text { Power: } 9 \times 200 \mathrm{~W}=1800 \mathrm{~W} \\
\text { Maximum temperature: } 190^{\circ} \mathrm{C}\end{array}$ \\
\hline 4 & Heat pad controller & Type: ESP 8266 NodeMCU \\
\hline
\end{tabular}

The second setup is shown in Fig. 2 and mainly differs in the measurement device with respective measurement points.

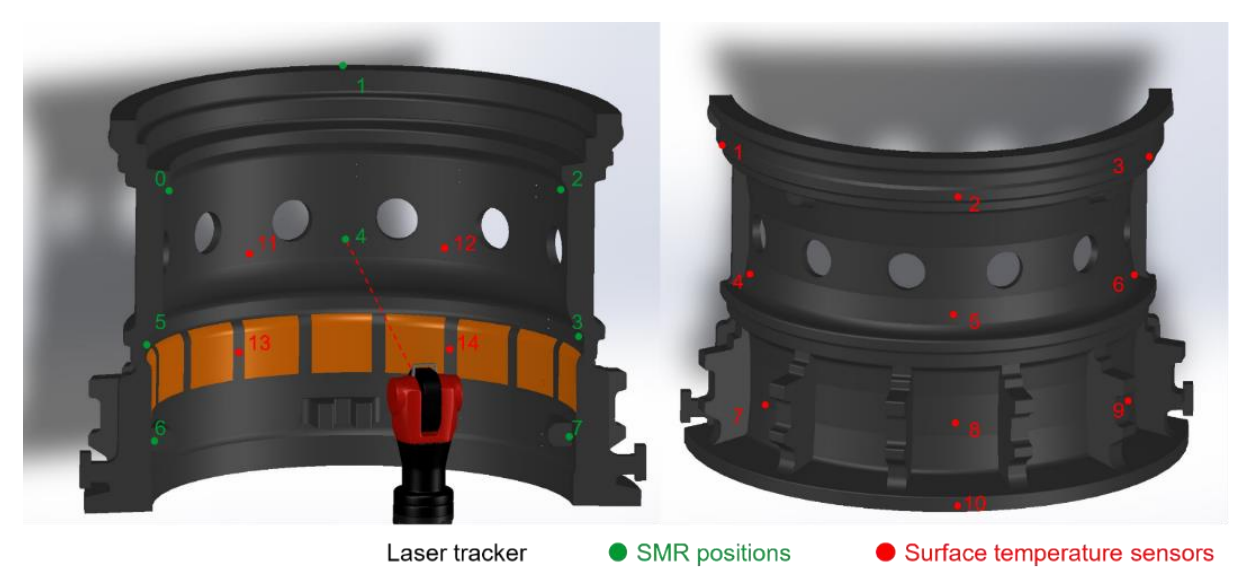

Fig. 2. Sensor and SMR positions in the laser tracker workpiece experiment 
Instead of the manually operated measurement arm an API Radian laser tracker is used to measure the center positions of 8 spherically mounted retroreflectors (SMRs), see green dots in Fig. 2. Red marked dots highlight surface temperature sensors.

\subsection{HEAT GENERATION UNIT}

Ensuring that heat generated deformations are detectable with available measurement systems, a proof of suitability is given with respect to chosen heat sources. As a conservative estimate given by the authors, the theoretical expected thermal expansion should exceed the maximum measurement uncertainty at least by factor 5, representing a $c_{\mathrm{pk}}$ level of 1.67. Thus, a probability of less than $1 \mathrm{ppm}$ for measuring wrong length differences due to dispersion of the metrology system can be inferred. Consequently, a preliminary rough estimation is calculated for the inner diameter. The analysis was done for the laser tracker measurements, where slightly higher uncertainties can be expected than for the mCMM according to manufacturer information:

$$
5 \cdot U<\emptyset_{\text {inside }} \cdot \Delta T \cdot C T E
$$

where: $U$ - measurement uncertainty of laser tracker, $C T E$ - coefficient of thermal expansion.

It should be noted that preliminary, empirical determined uncertainties for repeated distance measurements were smaller than provided manufacturer information (Fig. ) for both utilized measurement systems ( $\left.U_{\max }<10 \mu \mathrm{m}<U_{\text {MPE}}\right)$. This is due to anisotropic and task specific behavior of metrology systems. Taking that into account, detectable thermoelastic deformation can be assumed for a temperature difference of at least $3 \mathrm{~K}$ using (1). The power of heat pads must provide the necessary heat flux:

$$
P>A \cdot \Delta T \cdot \alpha
$$

where: $P$ - introduced power, $A$ - workpiece surface, $\Delta T$-aimed temperature difference, $\alpha$ - heat transfer coefficient

For heat loss due to convection and radiation a literature-based heat transfer coefficient of $\alpha=\alpha_{\text {con }}+\alpha_{\text {rad }}=18 \frac{\mathrm{W}}{\mathrm{m}^{2} \mathrm{~K}}$ is assumed. Taking mentioned variables into account, a power of at least $420 \mathrm{~W}$ is required to generate the envisaged temperature difference. Hence, the chosen setup of 9 heat pads will exceed calculated minimum power by factor 4 on the assumption of small loss.

\section{DESIGN OF EXPERIMENTS}

For physical investigation, two different experimental setups were realized, differing in testing time, collected measurement points, amount of temperature sensors, and utilized measurement systems, as introduced in 3.2. The main differences can be taken from Table 2 . 
Table 2. Comparison for mCMM and laser tracker setup

\begin{tabular}{|c|c|c|}
\hline & Setup $1-$ mCMM & Setup 2 - laser tracker \\
\hline Heat source & 9 heat pads, $200 \mathrm{~W}$ each & 9 heat pads, $200 \mathrm{~W}$ each \\
\hline Warm-up time & $190 \mathrm{~min}$ & $400 \mathrm{~min}$ \\
\hline Cool-down time & $290 \mathrm{~min}$ & $270 \mathrm{~min}$ \\
\hline $\begin{array}{c}\text { Measuring } \\
\text { strategy }\end{array}$ & $\begin{array}{c}\text { mode: manual } \\
\text { features: } 2 \text { distances } \\
\text { Collection: } 10 \text { repetitions per point } \\
\text { Measurement runs: } 5 \text { runs over } 360 \mathrm{~min}\end{array}$ & $\begin{array}{c}\text { mode: automatic } \\
\text { features: } 8 \text { distances } \\
\text { Collection: ca. } 3000 \text { repetitions per point } \\
\text { Measurement runs: } 336 \text { runs over } 770 \mathrm{~min}\end{array}$ \\
\hline $\begin{array}{c}\text { Temperature } \\
\text { sensors }\end{array}$ & 36 sensors & 14 sensors \\
\hline
\end{tabular}

\subsection{MCMM MEASUREMENT STRATEGY AND RESULTS}

The experiments with mCMM are conducted in a non-climatized environment, where drafts due to open doors, hall gates can occur. Before any test run, the measurement system is referenced over a dedicated set of points on the workpiece that build the local coordinate system. This decouples thermal effects from the workpiece to the mounted measurement system, which is relevant for absolute coordinate measurements, but has no effect on presented results, since only relative position changes are investigated. 5 measurement runs are collected over 6 hours -1 run for reference temperature, 2 runs during heating up and 2 runs during cooling down. For any measurement result, the mean value of 10 repeated measurements is determined.

In Fig. 3 the development of inner temperature distribution over time is illustrated.
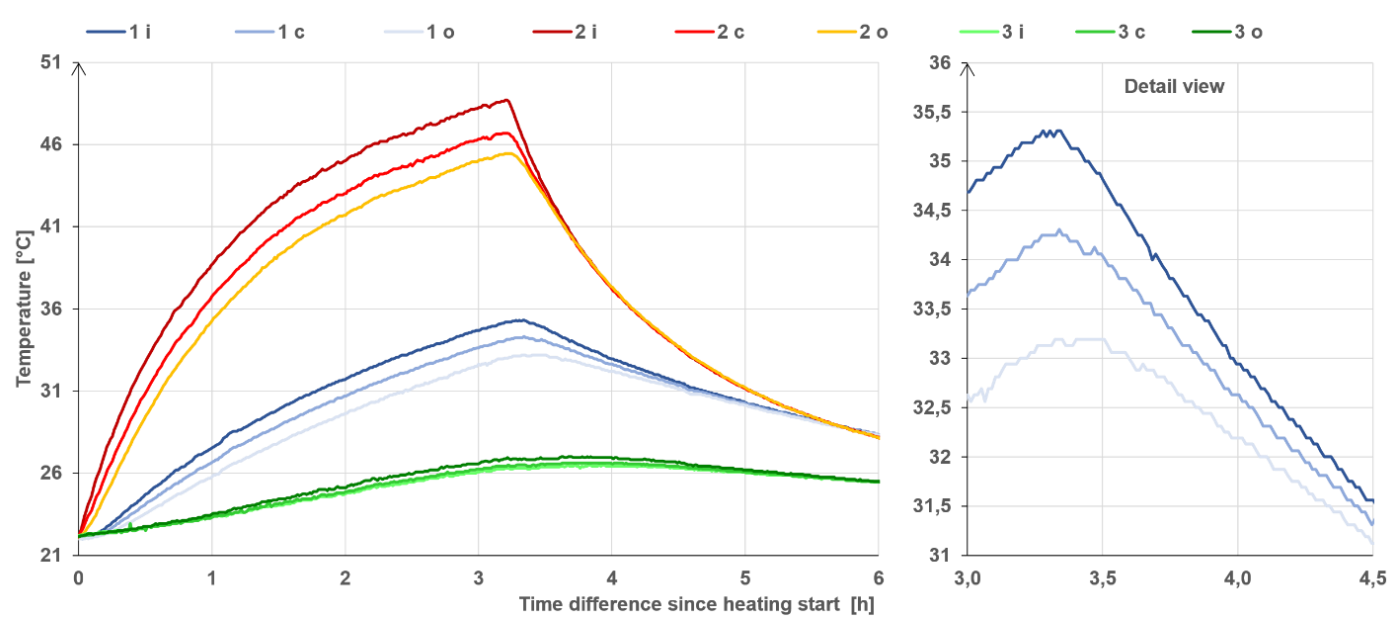

Fig. 3. Temperature over time for workpiece internal sensors. The sensors are radially distributed within the workpiece wall with sensors ,"i" located near the inner wall, „,c" in the center and „o" near the outer wall, see Fig. 1 for sensor positions

Especially for the middle, where sensors are more closely located to the heat sources, radial inhomogeneities of greater than $3 \mathrm{~K}$ are evident. The homogenization time during cooling down, depicted in detail view, reveals that after 60 minutes temperature differences 
of $1 \mathrm{~K}$ still occur, even though the workpiece can be considered as thin-walled part with high surface to mass ratio. For the bottom and top section, where sensors are mounted further from the heat pads, temperature differences over wall cross-sections can be clearly observed as well, but in smaller amplitudes. Thus, it is demonstrating an additional issue with compensation based on surface temperature measurements.

As described in Table 1, a total of 36 temperature sensors was utilized. For the purpose of transparency, solely surface sensor measurements close to the measurement points B1, B13 and B24 are presented. The results for geometric measurements and temperatures over time are depicted in Fig. 4, where red and blue backgrounds imply heating and cooling cycles respectively.

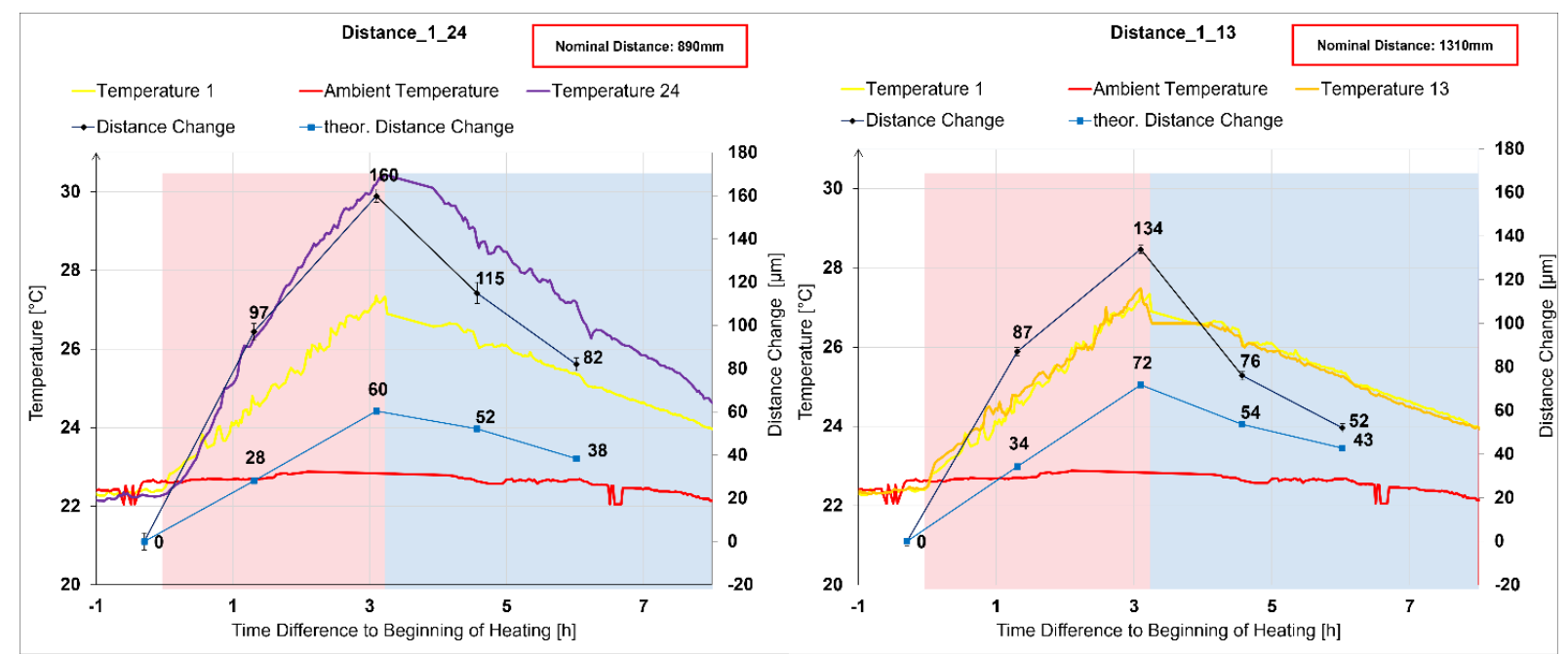

Fig. 4. Measured versus theoretical distance changes over time and respective temperatures including standard deviations for each point amplified by factor 2 for better visibility

As expected, the workpiece deforms due to temperature. For distance 1_24 a maximum deformation of $160 \mu \mathrm{m}$ can be observed, which is reached after 190 mins. Based on linear thermal expansion, a maximum deformation of $60 \mu \mathrm{m}$ is expected in contrast. At the same point of time a maximum deformation of $134 \mu \mathrm{m}$ can be observed for distance 1_13, whereby a calculated deformation of $72 \mu \mathrm{m}$ is expected. The mean temperature of the two closest sensors is considered in each case. It is striking that deviations between the measured and expected data are higher for distance 1_24 than for 1_13, despite a smaller nominal distance and only slightly elevated mean temperatures. This indicates that linear thermal models not just clearly undercut the measured deviations in presented case, but also that anisotropic expansion behaviour of the workpiece is evident.

\subsection{LASER TRACKER MEASUREMENT STRATEGY AND RESULTS}

With the data showing dynamic changes especially when switching off the heat influx (see Fig. 5), higher temporal resolution measurements are desirable. With the automated measurement setup data acquisition is possible in a faster frequency, facilitating equally high 
frequency time dependent dimensional evaluation. Besides, manual operator influences on the measurements can be circumvented.

The graphs in Fig. 5 show the correlation between different measured dimensions on the workpiece and different temperature values. The gray line shows linear thermal expansion assuming $11.1 \mathrm{ppm} / \mathrm{K} \mathrm{CTE}$, a literature value for $\mathrm{C} 45 \mathrm{E}$.

Relative position changes of the measurement points are depicted as the colored lines in Fig. 6 with an exaggeration factor of 1000 .

The colored dots show measurement values according to different temperature values.

There are three different color scales signifying time and differentiating the respective temperature values. The dots colored according to color scale 1 show the temperature measured at the center of the turbine housing, building an analogy to compensation of thermoelastic workpiece deformation with a single temperature (see Fig. 2: Sensor 5). For color scale 2 the mean value over all presumably relevant sensors was used as temperature input; e.g. for the mid diameter value the vertically middle sensors were used (see Fig. 2: Sensor 4-6), for the lower vertical distance on the right-side sensors 9 and 6 were used etc. For color scale 3 the mean value over all installed temperature sensors is used.
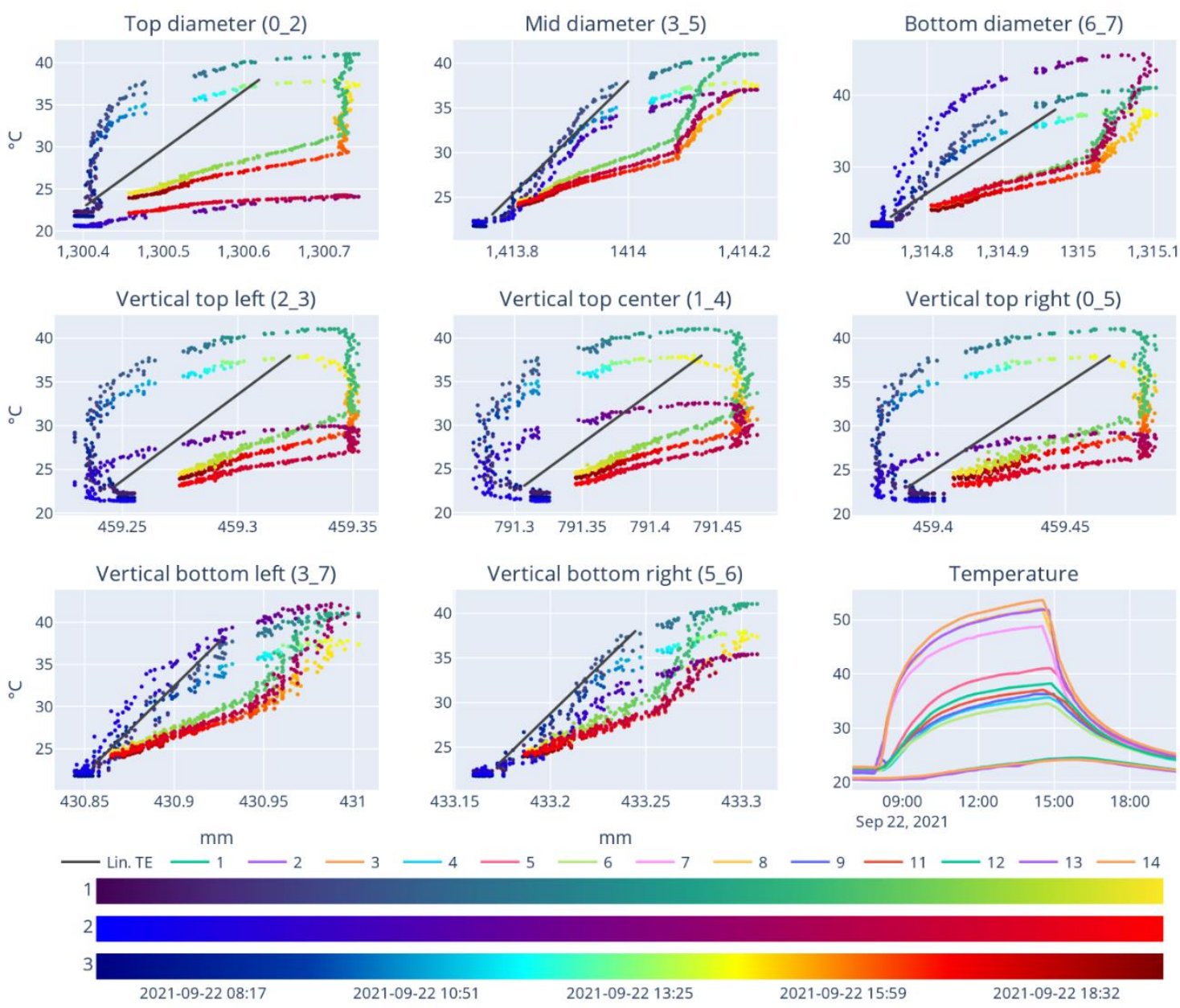

Fig. 5. Resulting data from the automated experiment. The graphs show the temperatures [y-axes] over the measured distances [x-axes]. The different color scales signify different temperature data sources. 1 - a single temperature sensor in the center of the workpiece; 2 - multiple temperature sensors close to the measurement positions; 3 - mean temperature over all part sensors. The gray line ("Lin. TE") shows linear thermal expansion) 
Analyzing the resulting data, the following takeaways can be inferred:

While thermal compensation is shown to generally improve measurement results, the experimental results clearly demonstrate issues with temperature compensation methods used for large scale metrology tasks. In inhomogeneous and/or transient thermal workpiece states thermoelastic deformation compensation based on a single temperature sensor as well as based on mean values over multiple temperature sensors is not sufficient, especially for larger features.

For diameter and shoulder measurements close to the heated area, the linear thermal model follows real measurement data generally closer (3_5, 6_7, 3_7, 5_6). For measurements further away from heated areas a lag and resulting hysteresis between temperature increase and decrease and dimension change can be seen $\left(0 \_2,2 \_3,1 \_5,0 \_5\right)$. The "top vertical" measurements exhibit a decrease in measured distance in the beginning, which the authors cannot explain, yet. When using averages over multiple sensors, some of which are closer to heated areas than the measurement position itself, temperature increase before measured distance increase is expected as can be seen in the "top" measurements.

Overall, for diameter measurements the measured distance increase is up to twice the expected diameter change due to linear thermal expansion (see Fig. 3 and Fig. 6).

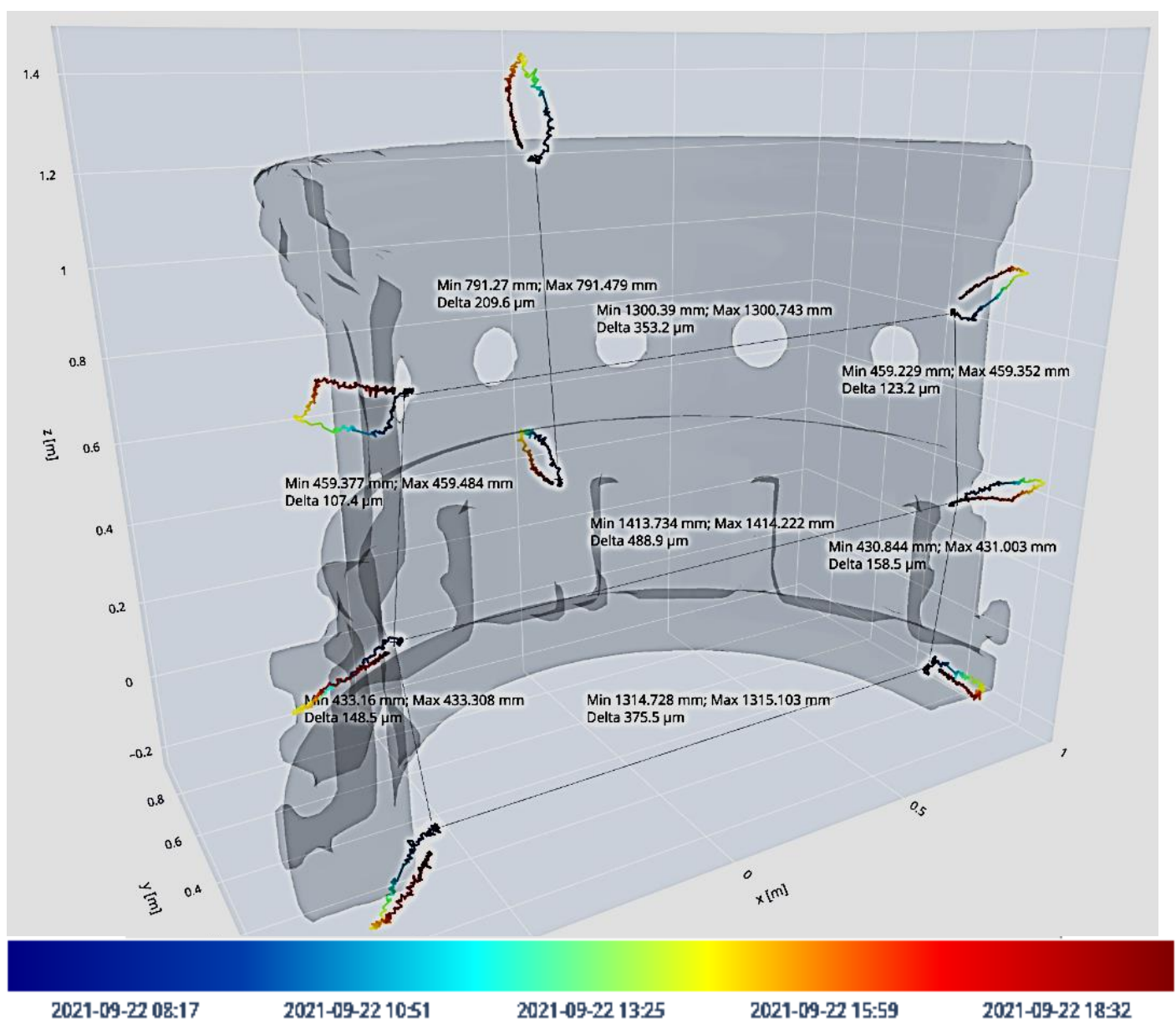

Fig. 6. SMR position change over time. The lines and text annotations show the measurements. The colored lines show the point positions over time with the relative change to the initial point measurement multiplied by a factor of 1000 


\section{CONCLUSIONS AND OUTLOOK}

The article presents an experimental setup to quantify transient thermoelastic effects for a large workpiece in a non-climatized environment. It consists of an exemplary turbine housing workpiece, electric and digitally controlled heat pads allowing simulated turning process heat input, temperature sensors, and 3D measurement systems. Two experiments were conducted: First a manual experiment with a mCMM and a second fully automated experiment using a laser tracker, to allow for higher temporal resolution measurements.

Both experiments showed thermoelastic deformation of up to more than two times the deformation calculated with the assumption of homogeneous temperature and linear thermal expansion - $160 \mu \mathrm{m}$ for mCMM measurements with 190 minutes of heating and $490 \mu \mathrm{m}$ for laser tracker measurements with 400 minutes of heating. Besides, it could be indicated that local heat fluxes result in significant transient temperature inhomogeneities over the workpiece surface as well as wall-cross sections accompanied by non-negligible temperature equalization times. Additionally, a significant anisotropic expansion behavior considering different measurement positions over the workpiece could be observed.

To compensate thermoelastic deformation more accurately, a higher fidelity thermoelastic model in FEM is advised. A digital twin of the setup using Ansys Software was already implemented. Due to lack of accuracy for the first prototype and scope restrictions, the authors forego a detailed presentation on results it this paper. It should be mentioned though that a high error sensitivity on assumed boundary condition parameters (e.g. heat flux, CTE, heat transfer coefficients) could be observed, making an a priori estimation based application of FEM unsatisfactory from a cost-benefit consideration as already discussed in Chapter 2 .

Nevertheless, future work will focus on further exploitation of the test rig in terms of laser tracker measurement series, which allow flexible and automized remote measurements over extended periods and more measurement points. Moreover, thermographic measurements are planned to increase resolution of thermal surface monitoring.

For better predictability, building a digital twin in FEM with subsequent model optimization will be next object of investigation. For instance, a feedback loop of real measurements, e.g. ambient temperatures and flow conditions to determine heat transfers, using CFX, is planned. Besides, parametric simulations with varying simulated boundary settings will be investigated aiming to find the necessary parameters to empirically match simulation results with real measurements.

Inverse modelling methods can be expedient to determine the introduced, unknown heat flux, which is currently estimated based on technical specifications and a priori assumptions. The authors conducted research in that regard using physics guided neural networks (PGNNs) to efficiently solve inverse heat transfer problems for simple geometries and will continue to do so for presented turbine housing [23] Subsequently, in a consistent approach at shopfloor measurements or on-machine measurements, the necessity for holistic compensation of not just machine errors but also workpiece deformation is evident. Therefore, a holistic consideration and error model fusion is mandatory as already advised by the authors in $[5,24]$. 


\section{ACKNOWLEDGEMENTS}

Funded by the Deutsche Forschungsgemeinschaft (DFG, German Research Foundation) under Germany's Excellence Strategy - EXC-2023 Internet of Production - 390621612 and - AutoKompII 298597595.

\section{REFERENCES}

[1] RAUCH M., HASCOET J.-Y., ROUSSEAU C., RUCKERT G., 2021, Thermal Monitoring for Metallic Additive Manufacturing Multi-Beads Multi-Layers Parts, Journal of Machine Engineering, 21/3, 92-100.

[2] GRZESIK W., 2020. Modelling of Heat Generation and Transfer in Metal Cutting: A Short Review, Journal of Machine Engineering, 20/1, 24-33.

[3] BEHRENS B.-A., BROSIUS A., HINTZE W., IHLENFELDT S., WULFSBERG J.P., 2021, Production at the Leading Edge of Technology, Springer Berlin Heidelberg, Berlin, Heidelberg.

[4] DEUTSCH J., ALBRECHT T., RIEDEL M., PENTER L., WIEMER H., MÜLlER J., IHLENFELDT S., 2020 , Thermo-Elastic Structural Analysis of a Machine Tool Using a Multi-Channel Absolute Laser Interferometer, Journal of Machine Engineering, 20/3, 63-75.

[5] DAHLEM P., EMONTS D., SANDERS M.P., SCHMITT R.H., 2020, A Review on Enabling Technologies for Resilient and Traceable on-Machine Measurements, Journal of Machine Engineering, 20/2, 5-17.

[6] YANG B., ROSS-PINNOCK D., MUELANER J., MULLINEUX G., 2017. Thermal Compensation for Large Volume Metrology and Structures, Int. J. Metrol. Qual. Eng. 8, 21, https://doi.org/10.1051/ijmqe/2017004.

[7] OHLENFORST M., 2019. Model-Based Thermoelastic State Evaluation of Large Workpieces for Geometric Inspection, RWTH Aachen University.

[8] ISO, 2013, Geometrical product specifications (GPS) - Coordinate measuring machines (CMM): Technique for determining the uncertainty of measurement - Part1: Overview and metrological characteristics (ISO 15530$1: 2013), 1$ st ed.

[9] ISO, 2009, Geometrical product specifications (GPS) - Acceptance and reverification tests for coordinate measuring machines (CMM) - Part 2: CMMs used for measuring linear dimensions (ISO 10360-2:2009).

[10] ISO, 2003, Geometrical product specifications (GPS) - Systematic errors and contributions to measurement uncertainty of length measurement due to thermal influences (ISO/TR 16015:2003).

[11] PAVLIČEK F., et al., 2015, Acclimatisation Time of Precise Workpieces for Quality Inspection, EUSPEN'S 15th International Conference \& Exhibition, Leuven, Belgium, June.

[12] ISO, 2011, Geometrical product specifications (GPS) - Coordinate measuring machines (CMM): Technique for determining the uncertainty of measurement - Part 3: Use of calibrated workpieces or measurement standards (ISO 15530-3:2011), 1st ed.

[13] ZELENY J., JANDA M., 2008, On-Machine Measurement Systems for High-Precision Workpieces, Produced on Five-Axis Milling Machines, Journal of Machine Engineering, 8/1, 11-18.

[14] PETEREK M., 2017, Messunsicherheitsbestimmung für Geometriemessungen mit Werkzeugmaschinen; 1. Auflage - Measurement uncertainty determination for geometry measurements using machine tools, Dissertation, Apprimus Verlag, Aachen, XV.

[15] ROSS-PINNOCK D., MULLINEUX G., 2018, Thermal Compensation Using the Hybrid Metrology Approach Compared to Traditional Scaling. Proceedings of the Institution of Mechanical Engineers, Part B: Journal of Engineering Manufacture, 232/13, 2364-2374.

[16] AKBAR F., MATIVENGA P.T., SHEIKH M.A., 2010, An Experimental and Coupled Thermo-Mechanical Finite Element Study of Heat Partition Effects in Machining, Int. J. Adv. Manuf. Technol., 46/5-8, 491-507.

[17] RAI J.K., XIROUCHAKIS P., 2009, FEM-Based Prediction of Workpiece Transient Temperature Distribution and Deformations During Milling, Int. J. Adv. Manuf. Technol., 42/5-6, 429-449.

[18] SCHINDLER S., ZIMMERMANN M., AURICH J.C., STEINMANN P., 2014, Finite Element Model to Calculate the Thermal Expansions of the Tool and the Workpiece in Dry Turning, Procedia CIRP, 14, 535-540.

[19] GLÄNZEL J., HERZOG R., IHLENFELDT S., MEYER A., UNGER R., 2016. Simulation-based Correction Approach for Thermo-elastic Workpiece Deformations During Milling Processes, Procedia CIRP, 46, $103-106$.

[20] DAW A., KARPATNE A., WATKINS W., READ J., KUMAR V., 2017, Physics-Guided Neural Networks (PGNN): An Application in Lake Temperature Modeling, http://arxiv.org/pdf/1710.11431v3.

[21] VDI Heat Atlas, 2010, Springer Berlin Heidelberg, Berlin, Heidelberg. 
[22] ISO, 2021, Statistical methods in process management - Capability and performance - Part 7: Capability of measurement processes (ISO 22514-7:2021).

[23] EMONTS D., YANG J., SCHMITT R.H., 2021, Solving Transient Inverse Heat Transfer Problems in Complex Geometries Using Physics-Guided Neural Networks (PGNN), MM SJ 2021, 3, 4540-4547.

[24] EMONTS D., SANDERS M.P., DAHLEM P., BODENBENNER M., MONTAVON B., SCHMITT H.R., 2021, Virtuelle Klimatisierung/Virtual Climatization, WT 111, 11-12, 887-892. 